\title{
Fe de erratas
}

César Román-Valencia, James. A. Vanegas-Ríos y Raquel I. Ruiz-C.

Especie nueva del género Bryconamericus (Teleostei: Characidae) del río Fonce, sistema río Magdalena, Colombia. Revista Mexicana de Biodiversidad 80(2), agosto: 455- 463, 2009.

Se presentan 4 Apéndices citados en el trabajo:

\section{Apéndice 1.}

Paratipos. IUQ 919, (61). COLOMBIA, departamento de Santander, municipio de Charalá, Sistema río Magdalena, cuenca río Fonce, Quebrada La Potrera, afluente Río Pienta, vía Charalá-La laguna (6 $10^{\circ} 42^{\prime \prime} \mathrm{N}$ y $\left.73^{\circ} 09^{\prime} 44^{\prime \prime}\right) 1457 \mathrm{msnm}, 5$ feb. 2004. IUQ 1569 (2) (C. \& T.). COLOMBIA, departamento Santander, municipio de Charalá, Sistema río Magdalena, cuenca río Fonce, Quebrada La Potrera, afluente Río Pienta, vía Charalá-La laguna (6 $10^{\prime} 42^{\prime \prime} \mathrm{N}$ y $\left.73^{\circ} 09^{\prime} 44^{\prime \prime}\right)$ 1457msnm, 5 feb. 2004. IUQ 921, (42). COLOMBIA, Departamento de Santander, municipio de Charalá, sistema río Magdalena medio, cuenca rio Fonce,

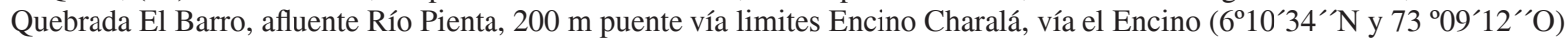
1457 msnm, 6 feb. 2004. IUQ 1573, (2) (C. \& T.). COLOMBIA, Departamento de Santander, municipio de Charalá, sistema río Magdalena medio, cuenca rio Fonce, Quebrada El Barro, afluente Río Pienta, 200 m puente vía limites Encino Charalá, vía el Encino (6 $6^{\circ} 10^{\prime} 34^{\prime} \mathrm{N}$ y $\left.73^{\circ} 09^{\prime} 12^{\prime \prime} \mathrm{O}\right) 1457$ msnm, 6 feb. 2004. IUQ 923, (36). COLOMBIA, Departamento de Santander, municipio de Charalá, sistema río Magdalena medio, cuenca río Fonce, Quebrada La Sanguina, afluente Río Pienta $\left(6^{\circ} 16^{\prime} 59^{\prime} \mathrm{N}\right.$ y $\left.73^{\circ} 09^{\prime 2} 21^{\prime \prime} \mathrm{O}\right)$ 1275 msnm, 5 feb. 2004. IUQ 1572 (2) (C. \& T.). COLOMBIA, departamento de Santander, municipio de Charalá, sistema río

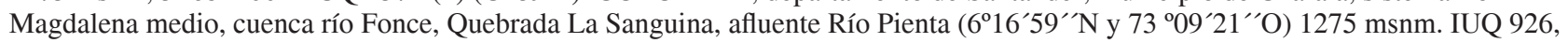
(21). COLOMBIA, departamento de Santander, municipio de Charalá, sistema río Magdalena medio, Río Fonce, afluente del Río San Gil, vía San Gil-Charalá (6 $6^{\circ} 27^{\prime} 00^{\prime} N$ y $73^{\circ} 08^{\prime} 51^{\prime \prime}$ O) 1198 msnm, 31 oct. 2003. IUQ 944 (2) (C. \& T.). Colombia, departamento de Santander, municipio de Charalá, sistema río Magdalena medio, río Fonce, afluente del Río San Gil, vía San Gil-Charalá (6²7’00`N y $73^{\circ} 08^{\prime} 51^{\prime \prime}$ O) $1198 \mathrm{msnm}, 31$ oct. 2003. IUQ 927 (33). COLOMBIA, departamento de Santander, municipio de Mogotes, sistema Magdalena medio, cuenca río Mogotes, Quebrada Guayawata, en el puente $100 \mathrm{~m}$ antes de su boca $\left(6^{\circ} 29^{\prime} 07^{\prime \prime} \mathrm{N}\right.$ y $\left.73^{\circ} 58^{\prime} 59^{\prime \prime} \mathrm{O}\right) 1^{1658}$ msnm, 1 nov. 2003. IUQ 937 (2) (C. \& T.). COLOMBIA, departamento de Santander, municipio de Mogotes, sistema magdalena medio, cuenca río Mogotes, Quebrada Guayawata, en el puente $100 \mathrm{~m}$ antes de su boca $\left(6^{\circ} 29^{\prime} 07^{\prime} \mathrm{N}\right.$ y $\left.73^{\circ} 58^{\prime} 59^{\prime \prime} \mathrm{O}\right) 1658 \mathrm{msnm}$, 1 nov. 2003. IUQ 932, (61). COLOMBIA, departamento de Santander, municipio de Mogotes, sistema río Magdalena medio, Río Mogotes, en la boca Quebrada Guayawata $100 \mathrm{~m}$ abajo del puente $1 \mathrm{Km}$ antes de Mogotes (6 $6^{\circ} 9^{\prime} 07^{\prime \prime} \mathrm{N}$ y $\left.73^{\circ} 58^{\prime} 59^{\prime \prime} \mathrm{O}\right) 1658 \mathrm{msnm}$, 7 feb. 2004. IUQ 1570 (2) (C. \& T.). COLOMBIA, departamento de Santander, municipio de Mogotes, sistema río Magdalena medio, Río Mogotes, en la boca Quebrada Guayawata $100 \mathrm{~m}$ abajo del puente $1 \mathrm{Km}$ antes de Mogotes $\left(6^{\circ} 29^{\prime} 07^{\prime \prime} \mathrm{N}\right.$ y $\left.73^{\circ} 58^{\prime} 59^{\prime \prime} \mathrm{O}\right)$ 1658 msnm, 7 feb. 2004. IUQ 1919, (77), colectados con el holotipo. AMNH 246523, (4), COLOMBIA, departamento de Santander, municipio de Mogotes, sistema río Magdalena medio, Río Mogotes, en la boca Quebrada Guayawata $100 \mathrm{~m}$ abajo del puente 1 $\mathrm{Km}$ antes de Mogotes (6 $6^{\circ} 29^{\prime} 07^{\prime \prime} \mathrm{N}$ y $\left.73^{\circ} 58^{\prime} 59^{\prime \prime} \mathrm{O}\right) 1658$ msnm, 7 feb. 2004. AMNH 246524, (5), COLOMBIA, departamento de Santander, municipio de Charalá, Vereda Los Chinitos, Sistema río Magdalena Medio, cuenca del río Fonce, Río Pienta vía a Encino aprox.10 km de Charalá $\left(6^{\circ} 13^{\prime} 00^{\prime} \mathrm{N}\right.$ y $\left.73^{\circ} 10^{\prime} 13^{\prime \prime} \mathrm{O}\right) 1658 \mathrm{msnm}, 17$ ene 2008. AMNH 246525, (2), COLOMBIA, departamento de Santander, municipio de Charalá, Sistema río Magdalena, cuenca río Fonce, Quebrada La Potrera, afluente Río Pienta, vía CharaláLa laguna $\left(6^{\circ} 10^{\prime} 42^{\prime \prime} \mathrm{N}\right.$ y $\left.73^{\circ} 09^{\prime} 44^{\prime \prime}\right) 1457 \mathrm{msnm}, 5$ feb. 2004.

Material de comparación examinado. B. alpha: (Véase Román-Valencia, 2003a). B. andresoi: IUQ 449 (véase Román-Valencia, 2003). B. bayano: IUQ 426, (9), PANAMÁ, cuenca río Bayano quebrada Cartí, afluente río Paja, $2 \mathrm{~km}$ aproximadamente vía Llano-San Blas (9 17' 16” N y 78 58' 40” O), 6 ago 1999. IUQ 1161, (2) (C. \& T., 41,8-45,35 mm LE), PANAMÁ, cuenca río Bayano quebrada Cartí, afluente río Paja 2, km aproximadamente vía Llano-San Blas $\left(9^{\circ} 17^{\prime} 16^{\prime}\right.$ N y $78^{\circ} 58^{\prime}$ 40” O), 6 ago 1999. B. brevirostris: MEPN 22 (1 de 2), El Oro, río Coluguro, 31 ago 1978. B. carlosi: (véase paratipos RománValencia, 2003c). B. caucanus: (Véase FMNH 56230 paratipos y IUQ 335, 336, 337, 340, 341, 342, 344, 346, 351, 352, 378, 379 en Román-Valencia, 2003a). MBUCV 27914, (15), COLOMBIA, Cesar, río Hurtado, afluente del río Cesar, cuenca del río Magdalena, sin fecha. IUQ 349, (113); COLOMBIA, Antioquia, Anorí; La Rosa, río Cauca; 17 oct 1988. IUQ 350, (77), COLOMBIA, La Guajira; río Ranchería; 24 ago 1981. IUQ 10, (4), COLOMBIA, Quindío, río La Vieja, quebrada afluente del río Quindío, 9 ago 1994. IUQ 227, (2), Valle, cuenca del río La Paila, 15 sep 1996. IUQ 254, (9), COLOMBIA, Quindío, río La Vieja en la balastrera, hacienda Playa Azul, jun 1996. IUQ 272, (1), Valle del Cauca, Alto Cauca, quebrada San Pablo, afluente río La Paila, 14 sep 1996 , IUQ 613, (4), COLOMBIA, Quindío, río La Vieja, vereda Maravélez, la Tebaida, 25 mar 1991. IUQ 1269, (3), COLOMBIA, Quindío, Río La Vieja, quebrada El Macho, afluente del río Barragán, 8 jul 1996. IUQ 1301, (6), COLOMBIA, Caldas, río Chamberri, afluente río Cauca, en la India, Salamina (5'24' 55” N y 75'30'10” O), 2 jul 2004. IUQ 856, (35), COLOMBIA, Antioquia, quebrada Los Dragos, afluente del río arriba vereda Francisca-Sacantin, Sonsón. IUQ 870, (32), COLOMBIA, Antioquia, río Anorí, afluente río Nechi, transecto vía Anorí-La Punta Anorí (7 4’ 17” N y 7507' 48” O a 1499 m), 10 sep 2003. IUQ 865, (8), COLOMBIA, Antioquia, quebrada La Chinca, afluente del río Anorí $200 \mathrm{~m}$ de La Punta, 10 sep 2003. IUQ 875, (38), COLOMBIA, Antioquia, quebrada La Tupia, afluente río Nechi, Anorí, 9 sep 2003. IUQ 862, (29), COLOMBIA, Antioquia, quebrada La Virgen, afluente río Anorí $100 \mathrm{~m}$ antes de su desembocadura, sector del río vía Anorí, La Punta Anorí (704'22” N y 7507'29” O a 1489 m). IUQ 872, (17), COLOMBIA, Risaralda, quebrada El Tigre, hacienda Guadalupe, vereda el Tigre, Pereira, 19 jul 2005. IUQ 859, (5), COLOMBIA, Antioquia, quebrada Serrana, afluente del río Anorí, 200 m de la vía Anorí, La Punta-Anorí, 10 sep 2003. IUQ 
858, (10), Antioquia, río Sonsón 100 m antes de la represa, Sonsón, jul 18 de 2004. IUQ 301b, (1) (C. \& T.), COLOMBIA, Quindío, alto río Quindío, alto Cauca, 28 ene 2000. IUQ 851, (6), río Anori, La Rosa, bajo Cauca, sistema río Magdalena, 17 oct 1988 . IUQ 861, (2) (C. \& T.), COLOMBIA, Antioquia, cuenca del Magdalena, quebrada La Virgen afluente Río Anorí, $100 \mathrm{~m}$ antes de su desembocadura, sector del río vía Anorí-La Punta (704’22” N y 7507’ 49” O) 1489 m, 10 sep 2003. IUQ 863, (2) (C. \& T.), COLOMBIA, Antioquia, cuenca del Magdalena, río Anorí afluente río Nechí, sector río vía Anorí-La Punta ( $7^{\circ} 4^{\prime} 17^{\prime}$ ' N y 75º $07^{\prime}$ 48” O) 1499 m, 10 sep 2003; IUQ 864, (2) (C. \& T.), COLOMBIA, Antioquia, cuenca del Magdalena, quebrada La Tupía afluente río Nechí, 9 sep 2003. IUQ 868, (2) (C. \& T.), COLOMBIA, Antioquia, cuenca del Magdalena, quebrada La Chinca afluente río Anorí, 200 m vía Anorí-La Punta, 10 sep 2003. IUQ 879, (1) (C. \& T.), COLOMBIA, Antioquia, cuenca del Magdalena, Quebrada Serrana afluente río Anorí, 200 m de la vía Anorí-La Punta, 10 sep 2003. IUQ 943, (1) (C. \& T.), COLOMBIA, Antioquia, río Sonsón $100 \mathrm{~m}$ antes de la represa, Sonsón, cuenca del Magdalena, 18 jul 2004. IUQ 948, (1) (C. \& T.), COLOMBIA, Antioquia, quebrada Los Dragos, afluente del río Arriba vereda Francisca-Sacantin, Sonsón, cuenca del Magdalena, 18 jul 2004. IUQ 1202, (1) (C. \& T.), COLOMBIA, Quindío, quebrada Boquia, afluente río Quindío. IUQ 1571, (2) (C. \& T.), Caldas, río Chamberri, afluente río Cauca, en la India, Salamina (5²4’ 55” N y 75³0’10” O). IUQ 1584, (1) (C. \& T.), COLOMBIA, La Guajira, río Ranchería, Caribe Colombiano, 20 ago 1981. B. cristiani: (véase Román-Valencia, 1998). B. dahli: (Paratipos, véase Román-Valencia, 2000). B. delta: MEPN 28, (11), ECUADOR, provincia Sucumbíos, Rio Tiputini, sector Mondaña 1km aguas abajo, 28 enero 1998. MEPN 29, (50 de 202), ECUADOR, provincia de Napo, río Huataracu frente a la comunidad, 12 feb 1995. MEPN 35, (40 de 85), ECUADOR, provincia de Sucumbíos, Rio Duguno, 2 km de la comuna Cofan, 1 may 1995. B. emperador: IUQ 375 (3), PANAMÁ, Río Calovebora. IUQ 321 (19), COLOMBIA, Chocó, municipio de Pizarro, cuenca río Piliza. B. exodon: DZSJRP 9088, (12), BRASIL, Mato Grosso, Santo António do Leverger, rio Cuiabá, próximo á foz do rio Aricá Mirim, divisa de Barão de Melgaço e Várzea Grande, 24 ene 2004. B. lambari: MCP 26057, (2), BRASIl, Rio Grande do Sul, Jacui, arroio Feitoria em Picada Verão (alf. Rio Cadeia, rio Caí, 02934'37'- 051 06' 15”), 26 jun 2000. B. pachacuti: MEPN 38, (20 de 51). ECUADOR, provincia de Morona-Santiago, río Yapapa afluente del río Santiago, 9 may 1991. B. heteresthes: IUQ 400, (3), COLOMBIA, Vaupés, sistema del río Amazonas, drenaje del lago Taraira, bajo río Apaporis. B. hypopterus: IUQ 398 (9), COLOMBIA, Caquetà, sistema Río Orteguaza, Quebrada Pompella sobre el puente vía Florencia-Belem, Caquetá. B. galvisi: (Paratipos véase Román-Valencia, 2000). B. gonzalezoi: IUQ 446 (véase paratipos Román-Valencia, 2002). IUQ 1168 (extraído de paratipos 446), (2) (C. \& T., 47,49-51,06 mm LE), PANAMÁ, río Bongie ( $9^{\circ} 21^{\prime} 35^{\prime \prime} \mathrm{N}$ y 82 36'35” O), 22 feb 1996. B. guizae: (Paratipos 451, véase Román-Valencia, 2003c). B. guaytarae: IUQ 348 (7), COLOMBIA, Departamento del Cauca, municipio del Patia, vereda La Manguita, cuenca del río Patia, $200 \mathrm{~m}$ debajo de la confluencia de los ríos Guachicono, San Bingo y San Jorge ( $1^{\circ} 56^{`} 19^{`} \mathrm{~N}$ y los $\left.77^{\circ} 08^{`} 23^{`} \mathrm{O}\right) 537 \mathrm{msnm}$. $\boldsymbol{B}$. huilae: (IUQ 423 en Román-Valencia, 2003d). IUQ 947, (2) (C. \& T.), COLOMBIA, Huila, quebrada La Criolla, afluente río Guachino, vereda Guachicos en la vía Pitalito-San Agustín, Alto Magdalena, 13 oct 2001. B. lambari: MCP 26057, (2), BRASIL, Rio Grande do Sul, Jacui, arroio Feitoria em Picada Verão (alf. Rio Cadeia, rio Caí, 029³4'37'- 051 06' $15^{\prime \prime}$ ), 26 jun 2000. B. loisae: (véase Román-Valencia, 2003a). B. multiradiatus: (véase Román-Valencia 2003a). B. meridae IUQ 393 (15),

VENEZUELA, estado Mérida, Rio Chama, puente No. 4 a 20 Km N-O de Mérida. B. iheringi: (véase Román-Valencia $2003 a$ ). $\boldsymbol{B}$. orinocoense: (Tipos véase Román-Valencia, 2003b). B. orteguasae: IUQ 408 (13), COLOMBIA, Putumayo, Orito, Río Orito en el puente vía a Caldero. B. pectinatus: MUSM 3825, dos ejemplares, PERÚ, Manu, P. N. M. Pakitza, quebrada Picaflor, 13 sep 1988. B. peruanus: IUQ 442 (25), COLOMBIA, Nariño, municipio de Tumaco, vertiente del Pacifico, afluente del río Caunapi, Quebrada Tangarial (1 ${ }^{\circ} 33^{\prime} 14^{\prime \prime} \mathrm{N}$ y los $78^{\circ} 41^{\prime} 25^{\prime}$ 'O) $30 \mathrm{~m}$. B. phoenicopterus: MEPN 40, (15 de 29), ECUADOR, provincia de MoronaSantiago, río Gualaquiza, 22 sep 1978. MEPN 44, (10 de 17), ECUADOR, provincia de Zamora-Chinchipe, río Nangaritza playa próxima destacamento Mayaycu, 18 jul 1993. B. plutarcoi: IaVH 3123, (12), COLOMBIA, Santander, río Suárez; ene 1994. IUQ 1577, (1) (C. \& T.), Santander, quebrada Santa Rosa de la desembocadura en el río Suárez $100 \mathrm{~m}$ hacia arriba, sistema del río Magdalena (6 26' 09' $\mathrm{N}$ y $\left.73^{\circ} 18^{\prime} 566^{\prime} \mathrm{O}\right) 825$ m, 24 jun 2001. B. scleroparius: (véase Román-Valencia, 2002). B. subtilisform: (Paratipos véase Román-Valencia 2003b). B. terrabensis: (véase Román-Valencia, 2002). B. thomasi: IUQ 1644 (3). ARGENTINA, sistema río Paraná, salta Orán, $4 \mathrm{Km}$ de aguas blancas, arroyo que cruza ruta 19. B. tolimae: (Ver IUQ 484, Román-Valencia, 2004). IUQ 1586, (2) (C. \& T.), COLOMBIA, Tolima, Ibagué, vereda Pastales, $100 \mathrm{~m}$ antes de Pastales en la vía Ibagué-Juntas, río Combeima, afluente río Magdalena (4³0’19” N y 75¹7’46” O) 1586 m, 27 dic 2002. B. yokiae: (Paratipos véase Román-Valencia, 2003e).

Apéndice 2. Frecuencias absolutas del conteo de escamas con poros en la línea lateral en poblaciones de B. foncensis sp. nov. Fonce, Mogotes y Pienta corresponden a los principales drenajes donde se distribuye la especie en la cuenca río Magdalena. N: tamaño de muestra, M: moda.

\begin{tabular}{lccccccccc} 
Población & $\mathrm{N}$ & 39 & 40 & 41 & 42 & 43 & 44 & 45 & $\mathrm{M}$ \\
Fonce & 22 & 5 & 5 & 12 & & & & & 41 \\
Mogotes & 93 & & 34 & 30 & 23 & 4 & 2 & & 40 \\
Pienta & 165 & 29 & 49 & 52 & 27 & 5 & 2 & 1 & 41 \\
\hline
\end{tabular}




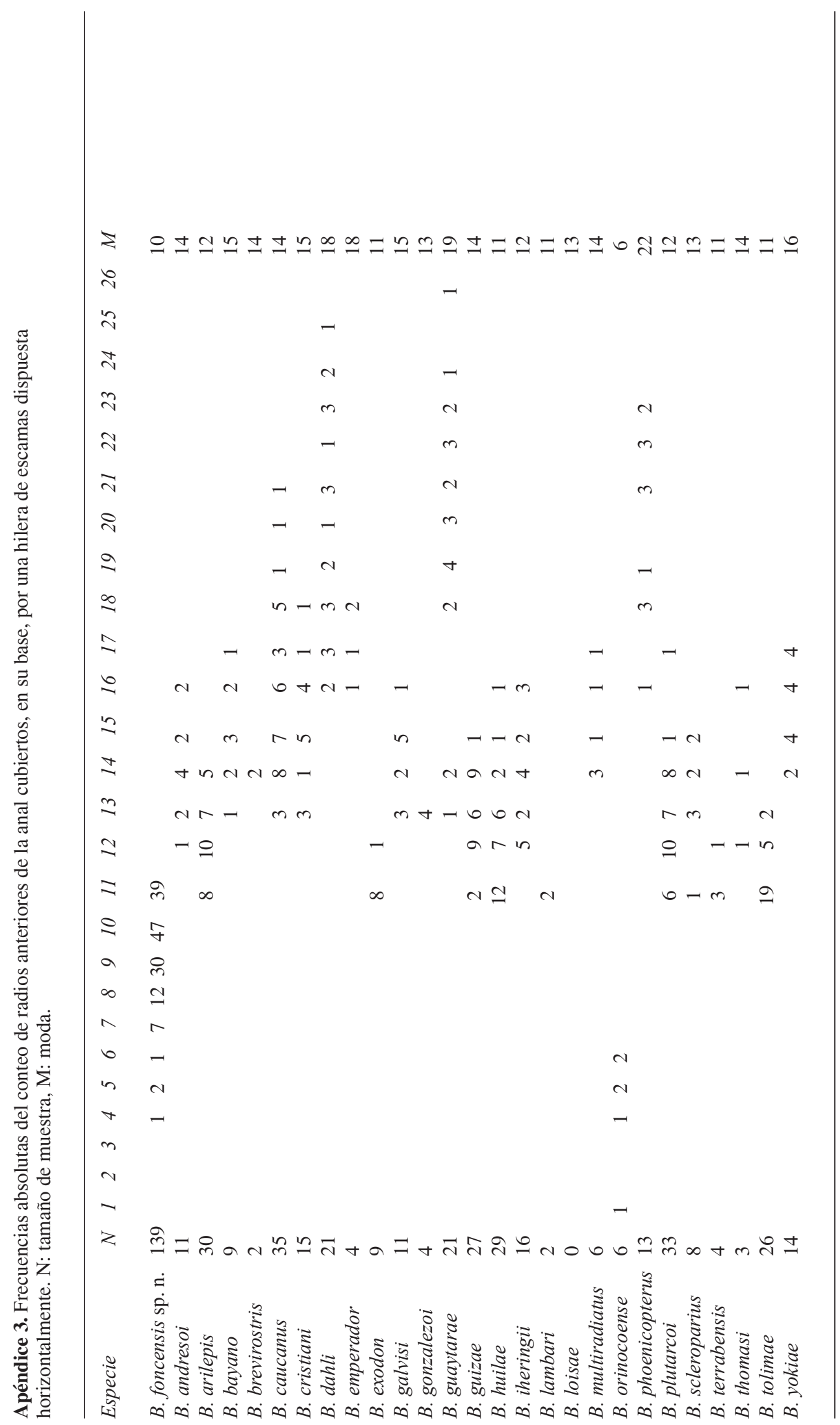


Apéndice 4. Frecuencias absolutas del conteo de escamas, ubicadas en hilera y horizontalmente, sobre la base de los primeros radios anteriores de aleta anal. N: tamaño de muestra, M: moda. N/A: no aplica.

\begin{tabular}{|c|c|c|c|c|c|c|c|c|c|c|c|c|c|c|c|c|c|c|c|c|c|c|c|c|}
\hline Especie & $\mathrm{N}$ & 1 & 2 & 3 & 4 & 5 & 6 & 7 & 8 & 9 & 10 & 11 & 12 & 13 & 14 & 15 & 16 & 17 & 18 & 19 & 20 & 21 & 22 & M \\
\hline B. foncensis sp n. & 151 & & & 4 & 4 & 17 & 28 & 26 & 36 & 21 & 11 & 4 & & & & & & & & & & & & 8 \\
\hline B. andresoi & 11 & & & & & & & & & & & 5 & 5 & 1 & & & & & & & & & & 12 \\
\hline B. arilepis & 29 & & & & & & & & & 2 & 8 & 11 & 5 & 3 & & & & & & & & & & 11 \\
\hline B. bayano & 9 & & & & & & & & & & & 4 & 2 & 1 & 1 & 1 & & & & & & & & 11 \\
\hline B. brevirostris & 2 & & & & & & & & & & & & 2 & & & & & & & & & & & 12 \\
\hline B. caucanus & 38 & & & & & & & & & & & & 15 & 11 & 6 & 4 & 1 & & & 1 & & & & 12 \\
\hline B. cristiani & 14 & & & & & & & & & & & & & 4 & 5 & 2 & 1 & 2 & & & & & & 14 \\
\hline B. dahli & 20 & & & & & & & & & & & & & & 4 & 3 & 2 & 5 & 3 & 1 & 1 & 1 & & 17 \\
\hline B. emperador & 25 & & & & & & & & & & & & 2 & & 2 & 7 & 3 & 2 & 6 & 1 & 1 & & 1 & 15 \\
\hline B. exodon & 11 & & & & & & & 3 & 3 & 3 & 2 & & & & & & & & & & & & & 9 \\
\hline B. galvisi & 11 & & & & & & & & & & & & 3 & 5 & 2 & & & 1 & & & & & & 13 \\
\hline B. gonzalezoi & 4 & & & & & & & & & 1 & 1 & 2 & & & & & & & & & & & & 11 \\
\hline B. guaytarae & 7 & & & & & & & & & & & 3 & 1 & 2 & 1 & & & & & & & & & 11 \\
\hline B. guizae & 28 & & & & & & & & & 1 & 12 & 6 & 7 & 2 & & & & & & & & & & 10 \\
\hline B. huilae & 34 & & & & & & & & 6 & 13 & 10 & 4 & 1 & & & & & & & & & & & 9 \\
\hline B. iheringii & 16 & & & & & & & & & & & 6 & 5 & 3 & 2 & & & & & & & & & 11 \\
\hline B. lambari & 2 & & & & & & & & & & 2 & & & & & & & & & & & & & 10 \\
\hline B. loisae & 2 & & & & & & & & & & & & 2 & & & & & & & & & & & 12 \\
\hline B. multiradiastus & 6 & & & & & & & & & & & 2 & 4 & & & & & & & & & & & 12 \\
\hline B. orinocoense & 6 & 3 & 3 & & & & & & & & & & & & & & & & & & & & & 1 \\
\hline B. phoenicopterus & 12 & & & & & & & & & & & & & & 2 & 1 & 2 & & 2 & 3 & & 2 & & 19 \\
\hline B. plutarcoi & 34 & & & & & & & & & & 9 & 16 & 3 & 4 & 2 & & & & & & & & & 11 \\
\hline B. scleroparius & 9 & & & & & & & & & & 1 & 3 & 2 & 3 & & & & & & & & & & 11 \\
\hline B. terrabensis & 4 & & & & & & & & & 1 & 3 & & & & & & & & & & & & & 10 \\
\hline B. thomasi & 3 & & & & & & & & & & 1 & 1 & & & 1 & & & & & & & & & N/A \\
\hline B. tolimae & 31 & & & & & & & & 4 & 3 & 11 & 9 & 4 & & & & & & & & & & & 10 \\
\hline B. yokiae & 11 & & & & & & & & & & & & 2 & 4 & 2 & & 2 & 1 & & & & & & 13 \\
\hline
\end{tabular}

